\title{
Associação entre o uso de prótese dentária total e o tipo de serviço odontológico utilizado entre idosos edêntulos totais
}

\author{
Association between the use of total dental prosthesis (denture) \\ and the type of oral health care service used by toothless elderly \\ individuals
}

Jairo Evangelista Nascimento (http://orcid.org/0000-0003-4010-3971) ${ }^{1}$ Tatiana Almeida de Magalhães (https://orcid.org/0000-0001-8371-863X) ${ }^{2}$ João Gabriel Silva Souza (https://orcid.org/0000-0001-5944-6953) ${ }^{3}$

Marinilza Soares Mota Sales (http://orcid.org/0000-0002-4987-3879) ${ }^{1}$

Charlitom Oliva Nascimento (https://orcid.org/0000-0001-6262-6824) ${ }^{4}$

Cláudio Wagnus Xavier Lopes Júnior (http://orcid.org/0000-0002-0358-0421) ${ }^{1}$

Efigênia Ferreira e Ferreira (https://orcid.org/0000-0002-0665-211X) ${ }^{5}$

Andréa Maria Eleutério de Barros Lima Martins (https://orcid.org/0000-0002-1205-9910) ${ }^{1}$

${ }^{1}$ Curso de Odontologia, Instituto de Ciências da Saúde, Faculdades Unidas do Norte de Minas (Funorte). Av. Osmani Barbosa 11.111, JK. 39404 006 Montes Claros MG Brasil.

jairomenmoc@gmail.com ${ }^{2}$ Programa de PósGraduação em Ciências da Saúde, Universidade Estadual de Montes Claros. Montes Claros MG Brasil.

${ }^{3}$ Faculdade de Odontologia de Piracicaba, Universidade Estadual de Campinas. Piracicaba SP Brasil.

${ }^{4}$ Funorte. Montes Claros MG Brasil.

${ }^{5}$ Departamento de Odontologia Social e Preventiva, Universidade Federal de Minas Gerais. Belo Horizonte MG Brasil.

\begin{abstract}
Oral rehabilitation with dental prosthesis was mandated by national oral health policy in Brazil, due to the high prevalence of total teeth loss and low use of dental prosthesis among the elderly. There is a pressing need to assess the impact of this rehabilitation policy. An attempt was therefore made to investigate if the dental service used was one of the factors associated with the use of total dental prosthesis among the elderly. To achieve this, a cross-sectional study using a probabilistic sample of elderly (60 years and above) urban-area residents of a small city was conducted by qualified examiners. Descriptive, bivariate and multiple (logistic regression/odds ratio - OR) analyses were conducted on 287 elderly people, of which 186 (64.8\%) used dental prosthesis. The use of total dental prosthesis was associated with a higher probability of being attended by private dental services $(O R=4.19 ; p<0.001)$ and the presence of lesions on the palate $(O R=7.52 ; p=$ 0.002). Conversely, it was associated with lower probability in ages greater than or equal to 73 years $(O R=0.52 ; p=0.023)$ and among those with impairment of OHIP-14 (OR $=0.20 ; p=$ 0.002 ) in the physical disability dimension. The use of total dental prosthesis was associated with the type of dental service used by the elderly, it being greater among private service users.
\end{abstract}

Key words Elderly, Oral health, Denture
Resumo A reabilitação protética foi prevista pela política nacional de saúde bucal em função da alta prevalência de perda de todos os dentes e do baixo uso de prótese dentária entre idosos. Há necessidade de se avaliar o impacto dessa política de reabilitação. Portanto, objetivou-se investigar se o tipo de serviço odontológico utilizado foi um dos fatores associados ao uso de prótese dentária total entre idosos. Trata-se de um estudo transversal conduzido em uma amostra probabilística de idosos (60 anos ou mais) residentes na zona urbana de um municipio brasileiro de pequeno porte populacional por examinadores calibrados. Realizaram-se análises descritiva, bivariada e múltipla (Regressão logística - odds ratio - OR). Participaram 287 idosos, destes, 186 (64,8\%) faziam uso de prótese dentária total. O uso de prótese dentária total foi associado a maiores chances de uso de serviços odontológicos supletivos ou particulares ( $O R$ $=4,19 ; p<0,001)$ e a presença de lesão no palato $(O R=7,52 ; p=0,002)$ e, também, a menores chances em idades maiores ou iguais a 73 anos $(O R=0,52 ; p=0,023)$ e entre os com comprometimento na dimensão incapacidade física do OHIP-14 (OR =0,20; $p=0,002)$. O uso de prótese dentária total foi associado ao tipo de serviço odontológico entre idosos, sendo maior o uso entre usuários dos serviços supletivos ou particulares.

Palavras-chave Idoso, Saúde bucal, Prótese total 


\section{Introdução}

A redução nas taxas de mortalidade, o declínio nas taxas de natalidade e o aumento da expectativa de vida como consequência das melhorias na assistência à saúde contribuíram para o envelhecimento populacional ${ }^{1}$, até mesmo em países em desenvolvimento, como o Brasil ${ }^{2}$. Em conformidade com essas mudanças, observa-se uma transformação do perfil epidemiológico dos brasileiros $^{3}$, que causa um aumento das necessidades e demandas entre idosos no campo da saúde. Nesse sentido, nota-se um destaque para a necessidade de uma maior atenção à saúde bucal dessa população $0^{4}$. Fato este que nem sempre acontece ${ }^{5}$.

Os idosos brasileiros, em sua maioria, apresentam condições de saúde bucal precárias. Segundo dados do levantamento nacional de saúde bucal, realizado em 2010, mais da metade dos idosos brasileiros não possuíam nenhum dente natural na boca $(53,7 \%)^{6,7}$. Tal fato pode ser decorrente da herança de um modelo assistencial pautado em práticas mutiladoras, ou seja, um número excessivo de extrações ${ }^{8}$. Além disso, apesar das mudanças no modelo assistencial e evidentes melhorias nas políticas públicas de saúde bucal no Brasil, ainda se nota um baixo uso de serviços odontológicos entre idosos ${ }^{6}$. Possivelmente, estes fatos são decorrentes de uma resiliência dos idosos em aceitar suas condições de saúde bucal, mesmo sendo precárias ${ }^{9}$, aliados à herança de um modelo assistencial com pouca acessibilidade e tratamentos mutiladores ${ }^{10,11}$. Dessa forma, a inacessibilidade aos serviços odontológicos, assim como fatores relacionados à qualidade dos mesmos, pode prejudicar o acesso ao tratamento reabilitador protético, o qual poderia superar as deficiências ocasionadas pelo edentulismo ${ }^{12}$.

$\mathrm{O}$ uso de prótese dentária entre idosos brasileiros ainda é baixo ${ }^{6}$. Subentende-se que os serviços públicos ainda não se estruturaram para ofertar próteses, apesar da Política Nacional de Saúde Bucal prever a ampliação e a qualificação da atenção primária, mediante a inclusão desses procedimentos $^{13}$. Houve avanços na oferta de serviços odontológicos no Brasil, mas ainda é evidente a necessidade de melhorias no acesso e na qualidade dos serviços para impactar positivamente nas condições subjetivas e normativas de saúde bucal dos idosos brasileiros ${ }^{11,14}$, inclusive na oferta de serviços de reabilitação protética de qualidade, e consequentemente no uso de próteses dentárias ${ }^{11,14,15}$. Além disso, ao considerar a alta prevalência de perda de todos os dentes entre idosos brasileiros, nota-se uma alta deman- da por serviços de reabilitação, o que possivelmente pode levar a longos períodos de espera e a busca por serviços particulares. Portanto, ainda não está claro na literatura se estas melhorias e a descentralização na oferta de serviços odontológicos públicos estão associadas a um maior uso de próteses dentárias totais entre idosos. Nesse sentido, o objetivo deste estudo foi avaliar se o tipo de serviço odontológico utilizado foi um dos fatores associados ao uso de prótese dentária total entre idosos brasileiros.

\section{Metodologia}

Este estudo possui delineamento transversal e foi realizado a partir de um projeto de extensão com interface em pesquisa intitulado "Reabilitação protética: impacto na qualidade de vida dos idosos de Ibiaí - estudo longitudinal". Tal projeto ofereceu reabilitação bucal, com próteses dentárias totais, aos idosos ( $\geq 60$ anos de idade) residentes na zona urbana de Ibiaí, Minas Gerais, que apresentaram necessidade deste tipo de tratamento, incluindo reparo ou substituição das próteses quando fosse necessário. Sua efetivação ocorreu mediante parceria entre a Universidade Estadual de Montes Claros (Unimontes) e a Prefeitura do município e contou com apoio financeiro da Fundação de Amparo à Pesquisa de Minas Gerais (FAPEMIG).

Segundo dados do Instituto Brasileiro de Geografia e Estatística (IBGE), o município era de pequeno porte populacional, com 7.839 habitantes em 2010, dentre eles, 505 idosos com 60 anos ou mais domiciliados na zona urbana. Ibiaí, na época da investigação, possuía três equipes da Estratégia Saúde da Família (ESF) que faziam a cobertura de $100 \%$ do município.

Os critérios de inclusão neste estudo foram estar em boas condições de saúde geral, ter utilizado serviço odontológico, ser edêntulo total, ou seja, não possuir dente natural em ambas as arcadas, além de ter idade igual ou superior a 60 anos e residir na zona urbana de Ibiaí. Assim, para estimar uma amostra representativa de participantes que atendessem aos critérios de inclusão, considerou-se que, dentre o universo de 505 idosos ( $\geq 60$ anos) de Ibiaí, conforme estimativa do IBGE, $66 \%$ atenderia aos critérios de inclusão, pois estudo prévio, conduzido entre idosos do sudeste brasileiro, identificou que dentre os idosos que haviam utilizado serviços odontológicos, $66 \%$ eram edêntulos totais ${ }^{16}$, ou seja, dentre os 505, 333 atenderiam ao critério de inclusão. 
A prevalência de usos de prótese entre edêntulos totais considerada para estimativa da amostra foi de $82 \%$ conforme resultado previamente registrado $^{16}$. Foi feito um cálculo amostral para população finita $=333$, considerando um nível de significância de $95 \%$, erro amostral de $2 \%$ e estimativa de $82 \%$ de ocorrência dos eventos ${ }^{6,17}$. O resultado foi uma amostra de 270 participantes.

No projeto de extensão, o primeiro passo foi procurar por todos os idosos, com idade igual ou superior a 60 anos e residência na zona urbana do município, com o intuito de identificar aqueles que tivessem necessidade de confecção, reparo ou substituição de próteses dentárias totais. Para isso, foi solicitada das três equipes da ESF uma lista com os nomes e endereços dos idosos cadastrados, que atendiam a estes critérios. Esta lista serviu como orientação para busca ativa dos mesmos, entretanto todos os domicílios urbanos do município foram visitados, considerando a possibilidade de encontrar outros idosos os quais, que por algum motivo não estivessem cadastrados na ESF. Durante as buscas, aproveitouse para entrevistar e examinar os idosos encontrados, que atendiam aos critérios de inclusão.

Os dados foram coletados por 3 duplas (um avaliador e um anotador) de acadêmicos do $10^{\circ}$ período do curso de odontologia da Universidade Estadual de Montes Claros (Unimontes), sob supervisão do professor/pesquisador responsável. Previamente à coleta, os acadêmicos foram treinados e calibrados (Kappa $\geq 0,61)^{18,19}$ segundo proposta da Organização Mundial de Saúde (OMS) em $1997^{20}$. A coleta dos dados ocorreu no período de janeiro de 2011 a julho de 2012, quando foram realizadas entrevistas nas quais os pesquisadores liam para os pesquisados as perguntas e as possíveis respostas dos questionários semiestruturados. Foram também realizados exames da cavidade bucal, sob luz natural, com auxílio de espelho clínico e espátula de madeira ${ }^{20}$.

As entrevistas utilizaram o roteiro empregado no levantamento Epidemiológico de Saúde Bucal Brasileiro ${ }^{16}$, para caracterização socioeconômica, avaliação do acesso a serviços odontológicos e da autopercepção em saúde bucal dos idosos participantes; um instrumento para avaliar os impactos consequentes das desordens bucais (OHIP), versão validada no Brasil do OHIP-1421; e um questionário de qualidade de vida relacionada à saúde, SF-12, na sua versão validada no Brasil ${ }^{22}$.

A variável dependente foi informação sobre o uso de prótese dentária total (PT) nas arcadas superior e inferior, denominada "Uso de Prótese dentária Total" e classificada em: não usa PT; usa apenas PT superior; usa apenas PT inferior; usa PT superior e inferior. Para processamento das análises de associação, essa variável foi dicotomizada, (Não, Sim), sendo: Não (Não usa PT) - Não usa prótese dentária total nas duas arcadas; Sim (Usa apenas PT superior, Usa apenas PT inferior e Usa superior e inferior) - Usa alguma prótese dentária total em pelo menos uma das arcadas.

As variáveis independentes foram agrupadas baseadas no modelo teórico de Andersen e Davidson ${ }^{23}$. Tal modelo propõe que os determinantes primários da saúde bucal (características do ambiente externo e aqueles pessoais da população e sistema de atenção à saúde bucal) influenciam o comportamento (uso de serviços odontológicos e práticas pessoais de higiene bucal). Estes, por sua vez, influenciam os desfechos em saúde bucal ${ }^{23}$. Dessa forma, os grupos de variáveis foram: características socioeconômicas/demográficas, características dos serviços odontológicos utilizados, condições de saúde geral, condições subjetivas de saúde geral/qualidade de vida, condições normativas de saúde bucal e perfil do impacto consequente das desordens bucais (OHIP).

As características socioeconômicas/demográficas foram representadas pelas variáveis: Idade categorizada pela média ( $<$ média $\mathrm{e} \geq$ à média) obtida pela média da idade que foi coletada em anos completos; Estado civil (solteiro/viúvo/divorciado e casado/união estável) obtido pela categorização das resposta da pergunta "Qual o seu estado civil?"; Sexo (feminino, masculino) obtido pela pergunta "Qual o seu sexo?"; Raça (branco e amarelo/indígena/negro/pardo) obtida pela coleta da informação, Raça/Grupo étnico, que utilizou a classificação do IBGE, que leva em consideração a autodeclaração do indivíduo; e Anos de estudo / Escolaridade formal ( $\operatorname{sim} \geq 1$ ano de estudo e não) obtida pela categorização da escolaridade coletada em anos de estudo completos, sendo: $\operatorname{sim}(\geq 1$ ano de estudo completo) e não (0 - não estuda/nunca estudou).

As características dos serviços odontológicos utilizados foram representadas pelas variáveis: Tipo de serviço odontológico utilizado (público, plano de saúde/particular e nunca utilizou) obtida pela categorização das respostas de duas perguntas "Já foi ao dentista alguma vez na vida? Onde?"; e Acesso a informações sobre como evitar problemas bucais (sim e não) obtida através da pergunta "Recebeu informações sobre como evitar problemas bucais?".

As condições de saúde geral foram representadas pelas variáveis: Doença crônica (não e sim) obtida pela pergunta "Quais doenças um médico 
já disse que você tem?” cujas opções, nenhuma e alguma (coletar por escrito), foram categorizadas em: não (nenhuma) e sim (alguma); e Uso de medicamento (não, sim) obtido pela pergunta "Quantos medicamentos de uso diário prescritos pelo médico você faz uso?" coletada em números inteiros e categorizada em: não (0-zero) e sim ( $\geq 1)$.

As condições subjetivas de saúde geral/qualidade de vida foram representadas pelas variáveis: Domínio Mental (SF-12) (Satisfatório e Insatisfatório) e Domínio Físico (SF-12) (Satisfatório e Insatisfatório). Os Domínios Físico e Mental do SF12 foram avaliados a partir da versão validada no Brasil do 12-Item Short-Form Health Survey (s12) composto por 12 itens que considera a percepção do indivíduo em relação aos aspectos de sua vida nas quatro últimas semanas. O processamento das 12 questões, cujas opções de respostas estão em uma escala tipo Likert, foi realizada a partir da aplicação de um algoritmo próprio do questionário, sendo calculados os dois domínios: o Físico (Physical Component Summary ou PCS) e o Mental (Mental Component Summary ou MCS). Pontos de corte adotados para níveis satisfatórios do domínio Físico e do domínio Mental foram, respectivamente, 50 e $42^{22}$.

As condições normativas de saúde bucal foram representadas por apenas uma variável: lesão no palato (não, sim), obtida por meio da avaliação da cavidade bucal, considerando o local da lesão (palato) e o tipo de lesão, categorizada em: não = sem lesão; e sim = mácula, mancha, pápula, placa, vesícula, bolha, erosão, úlcera, nódulo e tumor.

O perfil do impacto consequente das desordens bucais foi avaliado por meio da presença do impacto nas sete dimensões do OHIP-14, representadas pelas sete variáveis: Dimensão 1 - Limitação funcional (não e sim); Dimensão 2 - Dor física (não e sim); Dimensão 3 - Desconforto psicológico (não e sim); Dimensão 4 - Incapacidade física (não e sim); Dimensão 5 - Incapacidade psicológica (não e sim); Dimensão 6 - Incapacidade social (não e sim); Dimensão 7 - Deficiência (não e sim). Foi utilizada a versão traduzida e validada para a língua portuguesa e cultura brasileira do Oral Health Impact Profile (OHIP-14), instrumento que possibilita avaliar os impactos consequentes das desordens bucais nas dimensões física e psicossociais da saúde bucal, com 14 questões que foram agrupadas duas a duas em 7 dimensões: limitação funcional, dor física, desconforto psicológico, incapacidade física, incapacidade psicológica, incapacidade social e desvantagem social ${ }^{21}$. Para cada pergunta do OHIP-14, os idosos foram convidados a responder sobre a situação da sua boca e dos seus dentes nos últimos seis meses. Para a codificação das respostas foi utilizada uma escala do tipo Likert, que considera a frequência de experiência do(s) impacto(s), como se segue: 0-nunca, 1-raramente, 2-às vezes, 3-repetidamente, 4-sempre. A prevalência do impacto é obtida pelas respostas "repetidamente" ou "sempre" em pelo uma das perguntas do OHIP- $14^{24}$. A partir da proposta de Locker e Quiñonez ${ }^{24}$, a prevalência do impacto (não e sim) foi avaliada em cada uma das 7 dimensões do OHIP-14. Assim, a ocorrência de resposta "repetidamente" ou "sempre", em pelo menos uma questão que compõe a dimensão, implicou impacto nessa dimensão.

As análises foram conduzidas com auxílio do programa SPSS versão 17.0 para Windows', sendo que os dados foram apresentados em frequências absolutas e relativas na análise descritiva. Em seguida, foram conduzidas análises bivariadas entre a variável dependente e variáveis independentes pelo Qui-quadrado de Pearson. As variáveis com valor- $p$ menor ou igual $20 \%$ nas análises bivariadas foram incluídas nas análises múltiplas. A análise múltipla foi realizada através de Regressão logística, sendo estimados os valores de odds ratio, intervalo de confiança de $95 \%$ e nível de significância de $5 \%$.

O estudo foi conduzido conforme os princípios éticos da Declaração de Helsinki contidos na Resolução $n^{\circ} .196 / 96$ do Conselho Nacional de Saúde, após aprovação pelo Comitê de Ética e Pesquisa da Universidade Estadual de Montes Claros. Os idosos que aceitaram participar assinaram um termo de consentimento livre e esclarecido.

\section{Resultados}

Após todos os domicílios da zona urbana de Ibiaí terem sido visitados, foram encontrados 287 ido$\operatorname{sos}(\geq 60$ anos) edêntulos totais. Todos eles foram entrevistados e avaliados quanto as condições de saúde bucal e foram incluídos neste estudo; em termos práticos, todos os idosos (dentre os 505 idosos que atendiam aos critérios de inclusão) participaram do estudo embora a amostra estimada fosse de 270 idosos (Figura 1). A média de idade dos idosos foi 73,0 $( \pm 8,9)$ anos e a mediana 72,0 anos. Estes valores próximos indicam que as idades estão bem distribuídas em torno da média. Para as análises, a idade foi categorizada em $<73,0$ anos (abaixo da média) e $\geq 73,0$ (igual ou acima da média). 


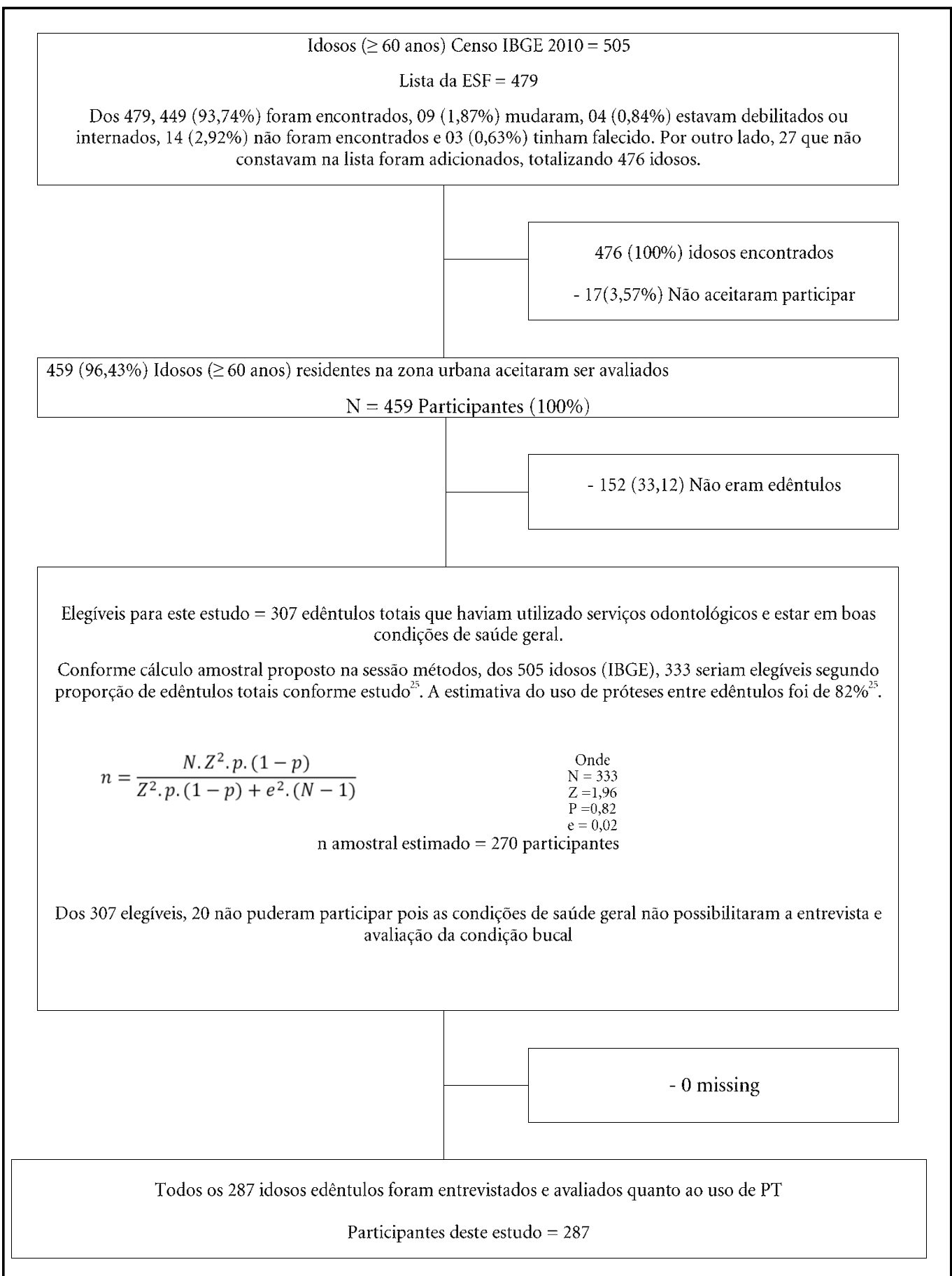

Figura 1. Fluxograma do número de participantes incluídos no estudo, a partir do inquérito conduzido entre idosos do município de Ibiaí-MG, realizado em 2011/2012.

A média de escolaridade em anos de estudos dos idosos foi de 1,6 $( \pm 2,24)$ anos, sendo que, 153 $(53,7 \%)$ idosos informaram não terem estudado e $271(95,1 \%)$ tinham até cinco anos de estudo, o que corresponderia, apenas, à $4^{\text {a }}$ série do antigo primário. Os idosos foram caracterizados quan- to às condições socioeconômico-demográficas, características dos serviços odontológicos utilizados, condições de saúde geral, condições subjetivas de saúde geral / qualidade de vida, condições normativas de saúde bucal e impacto consequentes das desordens bucais (OHIP-14) (Tabela 1). 
Tabela 1. Caracterização dos idosos quanto às variáveis investigadas em idosos de Ibiaí, Minas Gerais, 2011/2012. $(\mathrm{n}=287)$.

\begin{tabular}{|c|c|c|}
\hline Variáveis em estudo & $\mathbf{n}$ & $\%$ \\
\hline \multicolumn{3}{|l|}{ Uso de prótese dentária total } \\
\hline Não usa & 101 & 35,2 \\
\hline Usa apenas superior & 57 & 19,9 \\
\hline Usa apenas inferior & 1 & 0,3 \\
\hline Usa Superior e inferior & 128 & 44,6 \\
\hline \multicolumn{3}{|c|}{ Características socioeconômicas/demográficas } \\
\hline \multicolumn{3}{|c|}{ Idade categorizada (média $=73,0$ anos $)$} \\
\hline$<73,0$ anos & 149 & 51,9 \\
\hline$\geq 73,0$ anos & 138 & 48,1 \\
\hline \multicolumn{3}{|l|}{ Estado civil } \\
\hline Solteiro/Viúvo/divorciado & 153 & 53,3 \\
\hline Casado/união estável & 134 & 46,7 \\
\hline \multicolumn{3}{|l|}{ Sexo } \\
\hline Feminino & 183 & 63,8 \\
\hline Masculino & 104 & 36,2 \\
\hline \multicolumn{3}{|l|}{ Raça } \\
\hline Branco & 43 & 15,0 \\
\hline Amarelo/Indígena/Negro/Pardo & 244 & 85,0 \\
\hline \multicolumn{3}{|c|}{ Anos de estudo / Escolaridade formal ${ }^{*}-2$} \\
\hline $\operatorname{Sim} \geq 1$ ano de estudo & 132 & 46,3 \\
\hline Não & 153 & 53,7 \\
\hline \multicolumn{3}{|c|}{$\begin{array}{l}\text { Características dos serviços odontológicos } \\
\text { utilizados }\end{array}$} \\
\hline \multicolumn{3}{|c|}{ Tipo de serviço odontológico utilizado ${ }^{*}-4$} \\
\hline Público & 137 & 48,4 \\
\hline Plano de saúde /Particular & 129 & 45,6 \\
\hline Nunca utilizou & 17 & 6,0 \\
\hline \multicolumn{3}{|l|}{$\begin{array}{l}\text { Acesso a informações sobre como } \\
\text { evitar problemas bucais }{ }^{\star}-6\end{array}$} \\
\hline $\operatorname{Sim}$ & 106 & 37,7 \\
\hline Não & 175 & 62,3 \\
\hline \multicolumn{3}{|l|}{ Condições de saúde geral } \\
\hline \multicolumn{3}{|l|}{ Doença crônica } \\
\hline Não & 31 & 10,8 \\
\hline Sim & 256 & 89,2 \\
\hline \multicolumn{3}{|l|}{ Uso de medicamento ${ }^{*}-1$} \\
\hline Não & 66 & 23,1 \\
\hline Sim & 220 & 76,9 \\
\hline
\end{tabular}

continua

A Tabela 2 apresenta os resultados das análises bivariadas entre a variável dependente -uso de próteses totais - e as demais variáveis independentes. Dezesseis das vinte e três variáveis testadas apresentaram valor- $p \leq 0,20$ e foram consideradas nas análises múltiplas.
Tabela 1. Caracterização dos idosos quanto às variáveis investigadas em idosos de Ibiaí, Minas Gerais, 2011/2012. $(\mathrm{n}=287)$.

\begin{tabular}{lcc}
\hline \multicolumn{1}{c}{ Variáveis em estudo } & n & $\%$ \\
\hline Condições subjetivas de saúde geral/qualidade de vida \\
Domínio Mental (SF-12) ${ }^{*}-3$ & & \\
$\quad$ Satisfatório & 239 & 84,2 \\
$\quad$ Insatisfatório & 45 & 15,8 \\
Domínio Físico (SF-12) *-3 & & \\
$\quad$ Satisfatório & 127 & 44,7 \\
Insatisfatório & 157 & 55,3
\end{tabular}

\section{Condições normativas de saúde bucal}

Lesão no palato

$\begin{array}{lcc}\text { Não } & 251 & 87,5 \\ \text { Sim } & 36 & 12,5\end{array}$

Perfil do impacto da saúde bucal (OHIP)

Dimensão 1 - Limitação funcional ${ }^{*}-6$

$\begin{array}{lcc}\text { Não } & 260 & 92,5 \\ \text { Sim } & 21 & 7,5\end{array}$

Dimensão 2 - Dor física *-6

$\begin{array}{lcc}\text { Não } & 264 & 94,0 \\ \text { Sim } & 17 & 6,0\end{array}$

Dimensão 3 - Desconforto psicológico * -6

$\begin{array}{lcc}\text { Não } & 272 & 96,8 \\ \text { Sim } & 9 & 3,2\end{array}$

Dimensão 4 - Incapacidade física * 6

Não $260 \quad 92,5$

Sim $21 \quad 7,5$

Dimensão 5 - Incapacidade psicológica *-6

$\begin{array}{lll}\text { Não } & 261 & 92,9\end{array}$

Sim $\quad 20 \quad 7,1$

Dimensão 6 - Incapacidade social ${ }^{*}-6$

Não $276 \quad 98,2$

Sim $5 \quad \begin{array}{ll}1,8 \\ \end{array}$

Dimensão 7 - Deficiência * ${ }^{*} 6$

Não $\quad 275 \quad 97,9$

\begin{tabular}{crr} 
Sim & 6 & 2,1 \\
\hline $\mathrm{R} \$ 1,81=$ US $\$ 1,00$ dólar; ${ }^{*}$ Número de respondentes inferior
\end{tabular} a 287 .

$\mathrm{Na}$ análise múltipla identificou-se associação $(\mathrm{p} \leq 0,05)$ do uso de prótese dentária total com faixa etária, tipo de serviço odontológico utilizado, lesão no palato e impacto na incapacidade física (dimensão 4) do OHIP-14 (Tabela 3).

\section{Discussão}

O uso de prótese dentarias totais foi associado ao tipo de serviço odontológico utilizado pelo ido- 
Tabela 2. Análise bivariada dos fatores associados ao uso de próteses totais entre idosos de Ibiaí, Minas Gerais, 2011/2012. $(\mathrm{n}=287)$.

\begin{tabular}{|c|c|c|c|c|c|c|c|}
\hline \multirow{3}{*}{ Variável } & \multicolumn{4}{|c|}{ Uso de Prótese } & \multirow[b]{3}{*}{ OR } & \multirow[b]{3}{*}{ IC95\% } & \multirow[b]{3}{*}{$\mathbf{P}$} \\
\hline & \multicolumn{2}{|c|}{ Não } & \multicolumn{2}{|c|}{ Sim } & & & \\
\hline & $\mathbf{n}$ & $\%$ & $\mathbf{n}$ & $\%$ & & & \\
\hline \multicolumn{8}{|c|}{ Características socioeconômicas/demográficas } \\
\hline \multicolumn{8}{|c|}{ Idade categorizada (média = 73,0 anos) } \\
\hline$<73,0$ anos & 42 & 28,2 & 107 & 71,8 & 1,00 & & \\
\hline$\geq 73,0$ anos & 59 & 42,8 & 79 & 57,2 & 0,53 & $0,32-0,86$ & 0,010 \\
\hline \multicolumn{8}{|l|}{ Estado civil } \\
\hline Solteiro/Viúvo/divorciado & 55 & 35,9 & 98 & 64,1 & 1,00 & & \\
\hline Casado/União estável & 46 & 34,3 & 88 & 65,7 & 1,07 & $0,66-1,75$ & 0,774 \\
\hline \multicolumn{8}{|l|}{ Sexo } \\
\hline Feminino & 56 & 30,6 & 127 & 69,4 & 1,00 & & \\
\hline Masculino & 45 & 43,3 & 59 & 56,7 & 0,58 & $0,35-0,95$ & 0,031 \\
\hline \multicolumn{8}{|l|}{ Raça } \\
\hline Branco & 15 & 34,9 & 28 & 65,1 & 1,00 & & \\
\hline Não Branco & 86 & 35,2 & 158 & 64,8 & 0,98 & $0,5-1,94$ & 0,963 \\
\hline \multicolumn{8}{|c|}{ Anos de estudo / Escolaridade formal } \\
\hline $\operatorname{Sim} \geq 1$ ano de estudo & 36 & 27,3 & 96 & 72,7 & 1,00 & & \\
\hline Não & 64 & 41,8 & 89 & 58,2 & 0,52 & $0,32-0,86$ & 0,011 \\
\hline \multicolumn{8}{|c|}{ Características dos serviços odontológicos utilizados } \\
\hline \multicolumn{8}{|c|}{ Tipo de serviço odontológico utilizado } \\
\hline Público & 59 & 43,1 & 78 & 56,9 & 1,00 & & \\
\hline Plano de saúde /Particular & 24 & 18,6 & 105 & 81,4 & 3,31 & $1,9-5,78$ & 0,000 \\
\hline Nunca utilizou & 14 & 82,4 & 3 & 17,6 & 0,16 & $0,05-0,59$ & 0,006 \\
\hline \multicolumn{8}{|c|}{ Acesso a informações sobre como evitar problemas bucais } \\
\hline $\operatorname{Sim}$ & 32 & 30,2 & 74 & 69,8 & 1,00 & & \\
\hline Não & 65 & 37,1 & 110 & 62,9 & 0,73 & $0,44-1,23$ & 0,235 \\
\hline \multicolumn{8}{|l|}{ Condições de saúde geral } \\
\hline \multicolumn{8}{|l|}{ Doença crônica } \\
\hline Não & 13 & 41,9 & 18 & 58,1 & 1,00 & & \\
\hline $\operatorname{Sim}$ & 88 & 34,4 & 168 & 65,6 & 1,38 & $0,65-2,94$ & 0,407 \\
\hline \multicolumn{8}{|l|}{ Uso de medicamento } \\
\hline Não & 25 & 37,9 & 41 & 62,1 & 1,00 & & \\
\hline Sim & 76 & 34,5 & 144 & 65,5 & 1,16 & $0,65-2,04$ & 0,619 \\
\hline \multicolumn{8}{|c|}{ Condições subjetivas de saúde geral/qualidade de vida } \\
\hline \multicolumn{8}{|c|}{ Domínio Mental (SF-12) } \\
\hline Satisfatório & 78 & 32,6 & 161 & 67,4 & 1,00 & & \\
\hline Insatisfatório & 20 & 44,4 & 25 & 55,6 & 0,61 & $0,32-1,16$ & 0,129 \\
\hline \multicolumn{8}{|l|}{ Domínio Físico (SF-12) } \\
\hline Satisfatório & 37 & 29,1 & 90 & 70,9 & 1,00 & & \\
\hline Insatisfatório & 61 & 38,9 & 96 & 61,1 & 0,65 & $0,39-1,07$ & 0,088 \\
\hline \multicolumn{8}{|c|}{ Condições normativas de saúde bucal } \\
\hline Lesão no palato & & & & & & & \\
\hline Não & 98 & 39,0 & 153 & 61,0 & 1,00 & & \\
\hline Sim & 3 & 8,3 & 33 & 91,7 & 7,05 & $2,1-23,6$ & 0,002 \\
\hline
\end{tabular}

continua

so. Portanto, a chance do uso de próteses totais foi maior entre idosos que utilizaram serviços odontológicos particulares ou através de planos de saúde do que usuários de serviços públicos. 
Tabela 2. Análise bivariada dos fatores associados ao uso de próteses totais entre idosos de Ibiaí, Minas Gerais, 2011/2012. $(n=287)$.

\begin{tabular}{|c|c|c|c|c|c|c|c|}
\hline \multirow{3}{*}{ Variável } & \multicolumn{4}{|c|}{ Uso de Prótese } & \multirow[b]{3}{*}{ OR } & \multirow[b]{3}{*}{ IC95\% } & \multirow[b]{3}{*}{$\mathbf{P}$} \\
\hline & \multicolumn{2}{|c|}{ Não } & \multicolumn{2}{|c|}{ Sim } & & & \\
\hline & $\mathbf{n}$ & $\%$ & $\mathbf{n}$ & $\%$ & & & \\
\hline \multicolumn{8}{|c|}{ Perfil do impacto da saúde bucal (OHIP) } \\
\hline \multicolumn{8}{|c|}{ Dimensão 1 - Limitação funcional * } \\
\hline Não & 88 & 33,8 & 172 & 66,2 & 1,00 & & \\
\hline Sim & 10 & 47,6 & 11 & 52,4 & 0,56 & $0,23-1,38$ & 0,208 \\
\hline \multicolumn{8}{|l|}{ Dimensão 2 - Dor física * } \\
\hline Não & 89 & 33,7 & 175 & 66,3 & 1,00 & & \\
\hline Sim & 9 & 52,9 & 8 & 47,1 & 0,45 & $0,17-1,21$ & 0,115 \\
\hline \multicolumn{8}{|c|}{ Dimensão 3 - Desconforto psicológico * } \\
\hline Não & 92 & 33,8 & 180 & 66,2 & 1,00 & & \\
\hline $\operatorname{Sim}$ & 6 & 66,7 & 3 & 33,3 & 0,26 & $0,06-1,05$ & 0,058 \\
\hline \multicolumn{8}{|c|}{ Dimensão 4 - Incapacidade física * } \\
\hline Não & 85 & 32,7 & 175 & 67,3 & 1,00 & & \\
\hline Sim & 13 & 61,9 & 8 & 38,1 & 0,30 & $0,12-0,75$ & 0,010 \\
\hline \multicolumn{8}{|c|}{ Dimensão 5 - Incapacidade psicológica * } \\
\hline Não & 88 & 33,7 & 173 & 66,3 & 1,00 & & \\
\hline Sim & 10 & 50,0 & 10 & 50,0 & 0,51 & $0,2-1,27$ & 0,147 \\
\hline \multicolumn{8}{|c|}{ Dimensão 6 - Incapacidade social * } \\
\hline Não & 95 & 34,4 & 181 & 65,6 & 1,00 & & \\
\hline Sim & 3 & 60,0 & 2 & 40,0 & 0,35 & $0,06-2,13$ & 0,255 \\
\hline \multicolumn{8}{|c|}{ Dimensão 7 - Desvantagem social * } \\
\hline Não & 93 & 33,8 & 182 & 66,2 & 1,00 & & \\
\hline Sim & 5 & 83,3 & 1 & 16,7 & 0,10 & $0,01-0,89$ & 0,039 \\
\hline
\end{tabular}

Tabela 3. Análise múltipla dos fatores associados ao uso de próteses totais entre idosos de Ibiaí, Minas Gerais, 2011/2012. $(\mathrm{n}=280)$.

\begin{tabular}{llll}
\hline \multicolumn{1}{c}{ Variáveis } & OR & IC $_{95 \%}{ }^{*}$ & Valor-p \\
\hline Características socioeconômicas/demográficas \\
Idade categorizada \\
(média $=73,0$ anos) \\
$\quad$ & & & \\
$<73,0$ anos & 1,00 & & \\
$\geq 73,0$ anos & 0,52 & $0,29-0,91$ & 0,023
\end{tabular}

\section{Comportamento}

Tipo de serviço odontológico utilizado

$\begin{array}{lrll}\begin{array}{l}\text { Público } \\ \text { Plano de saúde / }\end{array} & 1,00 & & \\ \begin{array}{l}\text { Particular } \\ \text { Nunca utilizou }\end{array} & 0,19 & 2,28-7,68 & 0,000 \\ \text { Co, } & 05-0,75 & 0,017\end{array}$

\section{Condições normativas de saúde bucal}

Lesão no palato

\begin{tabular}{|c|c|c|}
\hline Não & 1,00 & \\
\hline Sim & 7,52 & \\
\hline
\end{tabular}

Perfil do impacto da saúde bucal (OHIP)

Dimensão 4 - Incapacidade física *

\begin{tabular}{|c|c|c|c|}
\hline Não & 1,00 & & \\
\hline Sim & 0,20 & $0,07-0,55$ & 0,002 \\
\hline
\end{tabular}

Hosmer-Lemeshow
Pseudo $\mathrm{R}^{2}=0,294$
A maioria dos idosos investigados fazia uso de prótese dentária total $(64,8 \%)$, esperava-se que este uso fosse maior, já que a amostra foi composta apenas por edêntulos totais. Portanto, espera-se que pessoas que não possuem nenhum dente na boca façam uso de próteses dentárias totais, principalmente ao considerar o fato de que as limitações impostas pelas perdas dentárias poderiam ser superadas pela reabilitação com próteses dentárias totais ${ }^{12}$. A prevalência identificada foi inferior à encontrada em uma amostra de idosos desdentados da região Sudeste brasileira oriunda do SB Brasil 2002/2003, onde o uso de prótese dentária em pelo menos uma arcada foi superior a $80 \%{ }^{25}$. Salienta-se ainda que a prevalência encontrada foi semelhante à prevalência registrada em estudos prévios realizados entre idosos dentados e desdentados em Porto Alegre $(\mathrm{RS})^{26}$ e Rio Claro (SP $)^{27}$, fato preocupante pois tal uso deveria ser maior. Nota-se, entretanto, que a amostra foi composta, principalmente, por idosos de baixa renda, o que pode ter limitado o acesso a serviços de reabilitação protética, possível explicação para prevalência encontrada. Tal 
fato é ainda mais evidente ao considerar que o uso de prótese foi maior entre usuários de serviços particulares ou de planos de saúde.

O tipo de serviço odontológico e a não utilização dos serviços mantiveram-se associados à reabilitação com próteses dentárias totais. Os idosos brasileiros foram prejudicados pela precariedade na oferta de serviços odontológicos e por uma prática odontológica mutiladora ${ }^{6,28}$. A reabilitação tem sido a única opção dos edêntulos totais, e por se tratar de um procedimento especializado, pode ser cara $^{29}$. Porém, problemas relacionados ao acesso aos serviços odontológicos públicos, assim como a longa espera por tratamentos especializados como os de prótese dentária podem levar os idosos a procurarem alternativas no serviço privado. As desigualdades sociais inviabilizam a acessibilidade ao tratamento reabilitador assim como inviabilizam o acesso a qualquer tipo de serviço odontológico. Dessa forma, destaca-se ainda a importância da estruturação de um modelo de vigilância em saúde em que o monitoramento de desigualdades no perfil de saúde bucal configure-se como um dos principais elementos ${ }^{30}$. Apesar da ampliação da oferta de serviços odontológicos públicos no Brasil, inclusive os especializados, a partir da política nacional de saúde bucal (Brasil Sorridente) e da incorporação da equipe de saúde bucal na $\mathrm{ESF}^{31}$, tal associação pode indicar que a população investigada ainda não foi adequadamente beneficiada. A investigação sobre onde foram confeccionadas as próteses ofertadas para os usuários da ESF não foi feita, fragilizando a associação registrada entre o tipo de serviço utilizado e o uso de próteses.

$\mathrm{O}$ uso de prótese dentária foi menor entre idosos mais velhos (mais de 72 anos). O edentulismo, visto como irreversível, leva o idoso a se conformar. Os sentimentos podem passar por estágios que vão desde negação, raiva, depressão, até adaptação e aceitação ${ }^{32}$. O idoso aceita sua precária saúde bucal, não acreditando que mereça ou que seja possível melhorá-la ${ }^{33}$. Portanto, o envelhecimento pode acarretar em conformação e resiliência em relação a condição de saúde bucal, fazendo com que o idoso não busque por melhorias, inclusive o uso de prótese dentária. No entanto, ressalta-se que o presente estudo não avaliou o uso prévio de prótese dentária, portanto, não se sabe se esses idosos mais velhos já utilizaram anteriormente.

A presença de lesão no palato esteve associada a um maior uso de prótese dentária entre os investigados. As próteses dentárias quando não são bem confeccionadas e/ou mal adaptadas podem causar lesões ${ }^{34}$, tais como estomatites, hiperplasia e queilite angular, o que eleva a prevalência deste tipo de lesão nestes usuários. Além disso, os usuários de prótese revelam ter dificuldades de adaptação e desconforto, e às vezes, continuam usando as próteses em função da aparência ${ }^{35}$, fator que pode desencadear a ocorrência de lesões. Em avaliação da prevalência de lesões dos tecidos moles causadas por próteses removíveis, em pacientes atendidos em clínica universitária de Pernambuco, constatou-se que 39,5\% dos pacientes apresentavam algum tipo de lesão causada pelo aparelho protético ${ }^{36}$. Ressalta-se que tais lesões podem gerar desconfortos físicos, psicológicos e estarem associadas à ocorrência de agravos bucais mais severos. Investigação prévia constatou que as lesões decorrentes do uso de prótese mal adaptadas estiveram associadas à ocorrência de carcinoma $^{37}$. Salienta-se a necessidade de considerar os princípios básicos do tratamento reabilitador, além disso, a falta de diálogo profissional/ paciente durante o tratamento impossibilita a identificação de problemas percebidos pelos idosos que devem ser considerados na reabilitação, afim de evitar tais lesões. No entanto, ressalta-se que no presente estudo não foi avaliado a qualidade das próteses dentárias. Em adição, deve se considerar também a possibilidade da existência deste tipo de lesão antes mesmo do tratamento reabilitador, podendo ser esta presença negligenciada pelo clínico. Além disso, a presença previa da lesão pode levar a não adaptação da prótese e abandono do uso. Portanto, tais fatores devem ser considerados no tratamento reabilitador.

As investigações realizadas na área de Odontologia, em sua maioria, buscaram avaliar as condições dentárias e tratamentos realizados, ignorando as experiências do paciente. No entanto, a condição clínica por si só não indica o quanto as pessoas se sentem afetadas pela sua condição bucal $^{38}$. Ressalta-se que a expressão "saúde bucal" aborda um conjunto de condições normativas e subjetivas, que possibilita ao ser humano praticar distintas funções, exercitar a autoestima e relacionar-se socialmente ${ }^{39}$. Além disso, impactos (consequências) decorrentes das desordens bucais estão associados ao comprometimento da qualidade de vida de idosos ${ }^{40}$. No presente estudo a dimensão incapacidade física do OHIP esteve associada a um menor uso de prótese dentaria total. Os idosos brasileiros, assim como os da presente investigação, possuem altas taxas de edentulismo ${ }^{41}$, fator este que pode influenciar a mastigação, dentre outras limitações funcionais. Ressalta-se que a falta de dentes pode gerar pro- 
blemas na fala, mastigação e na aparência com graves repercussões na autoestima e na socialização ${ }^{32}$. Ainda no campo das questões subjetivas, revisão sistemática da literatura apontou o impacto da perda dentária no comprometimento da qualidade de vida relacionada à saúde buca $l^{42}$. Portanto, o edentulismo associado com a não utilização de prótese dentária pode acarretar em impactos negativos nas condições físicas relacionadas à saúde bucal. Salienta-se ainda que estudo prévio demonstrou melhoria nos impactos decorrentes das desordens bucais em indivíduos que foram reabilitados com prótese dentária removível ${ }^{43}$, apontando que a reabilitação protética pode ter potencial em superar as incapacidades físicas geradas pela perda dentária.

Dentre as limitações do presente estudo, ressalta-se a não avaliação de questões relacionadas à reabilitação protética, como, por exemplo, o local de confecção da prótese, abandono do uso e, principalmente, a qualidade das próteses utilizadas. Salienta-se que alguns desses fatores não avaliados poderiam explicar melhor as associações identificadas. Apesar disso, a investigação foi conduzida com rigor metodológico necessário para estudos epidemiológicos identificando resultados relevantes que podem gerar subsídios para melhoria das políticas públicas de saúde bucal que visem a ampliação do acesso ao tratamento reabilitador e, consequentemente, melhorias das condições de vida desse estrato populacional.

Conclui-se que, o uso de prótese dentária total foi maior entre idosos que utilizaram serviços odontológicos particulares ou de planos de saúde. Portanto, o uso dessas próteses está associado ao tipo de serviço odontológico utilizado.

\section{Colaboradores}

JE Nascimento e AMEBL Martins participaram da concepção e desenho metodológico do estudo. JE Nascimento, TA Magalhães, MSM Sales, CO Nascimento e CWX Lopes Júnior participaram da revisão de literatura, da coleta de dados e da redação do artigo. JE Nascimento, AMEBL Martins, JGS Souza participaram da revisão da literatura, tratamento e análise dos dados e redação do artigo. AMEBL Martins conduziu a revisão final do artigo. EF Ferreira contribuiu na redação e na revisão final do manuscrito.

\section{Agradecimentos}

Agradecemos à Unimontes e a Prefeitura Municipal de Ibiaí a parceria que viabilizou o projeto. Agradecemos à FAPEMIG o apoio financeiro. Agradecemos aos acadêmicos que contribuíram na coleta de dados do projeto. Andréa Maria Eleutério de Barros Lima Martins foi bolsista de pós doutorado do CNPQ e bolsista de produtividade da FAPEMIG, e Efigênia Ferreira e Ferreira é bolsista de produtividade do CNPQ. 


\section{Referências}

1. Christensen K, Doblhammer G, Rau R, Vaupel JW. Ageing populations: the challenges ahead. Lancet 2009; 374(9696):1196-1108.

2. Veras R. Envelhecimento populacional contemporâneo: demandas, desafios e inovações. Rev Saude Publica 2009; 43(3):548-554.

3. Schmidt MI, Duncan BB, Azevedo e Silva G, Menezes AM, Monteiro CA, Barreto SM, Chor D, Menezes PR. Doenças crônicas não transmissíveis no Brasil: carga e desafios atuais. Lancet 2011; 377:2042-2053.

4. Ferreira RC, Vargas AMD, Fernandes NCN, Souza JGS, Sá MAB, Oliveira LFB, Martins AMEBL. O idoso com comprometimento cognitivo apresenta pior condição de saúde bucal? Cien Saude Colet 2014; 19(8):3417-3428.

5. Colussi CF, Freitas SFT. Aspectos epidemiológicos da saúde bucal do idoso no Brasil. Cad Saude Publica 2002; 18(5):1313-1320.

6. Brasil. Ministério da Saúde (MS). Secretaria de Atenção à Saúde. Departamento de Atenção Básica. Coordenação Nacional de Saúde Bucal. Projeto SB Brasil 2010: Condições de Saúde Bucal da População Brasileira, Resultados Principais. Brasília: MS; 2011.

7. Peres MA, Barbato PR, Reis SCGB, Freitas CHSM, Antunes JLF. Perdas dentárias no Brasil: análise da Pesquisa Nacional de Saúde Bucal 2010. Rev Saude Publica 2013; 47(Supl. 3):78-89.

8. Matos DL, Giatti L, Lima-Costa MF. Fatores sóciodemográficos associados ao uso de serviços odontológicos entre idosos brasileiros: um estudo baseado na Pesquisa Nacional por Amostra de Domicílio. Cad Saude Publica 2004; 20(5):1290-1297.

9. Martins AMEBL, Barreto SM, Pordeus IA. Autoavaliação de saúde bucal em idosos: análise com base em modelo multidimensional. Cad Saude Publica 2009; 25(2):421-435.

10. Moreira RS, Nico LS, Tomita NE, Ruiz T. A saúde bucal do idoso brasileiro: revisão sistemática sobre o quadro epidemiológico e acesso aos serviços de saúde bucal. Cad Saude Publica 2005; 21(6):1665-1675.

11. Martins AMEBL, Jardim LA, Souza JGS, Rodrigues CAQ, Ferreira RC, Pordeus IA. Is the negative evaluation of dental services among the Brazilian elderly population associated with the type of service? Rev Bras Epidemiol 2014; 17(1):71-90.

12. Ferreira AAA, Piuzevam G, Werner CWA, Alves MSCF. A dor e a perda dentária: representações sociais do cuidado à saúde bucal. Cien Saude Colet 2006; 11(1):211-218.

13. Brasil. Ministério da Saúde (MS). Saúde Bucal. Brasília: MS; 2006 .

14. Martins AMEBL, Barreto SM, Pordeus IA. Uso de serviços odontológicos entre idosos brasileiros. Rev Panam Salud Publica 2007; 22(5):308-316.

15. Baldani MH, Brito WH, Lawder JAC, Mendes YBE, Silva FFM, Antunes JLF. Determinantes individuais da utilização de serviços odontológicos por adultos e idosos de baixa renda. Rev bras Epidemiol 2010; 13(1):150-162.

16. Brasil. Ministério da Saúde (MS). Projeto SB Brasil 2003: condições de saúde bucal da população brasileira 2002-2003: resultados principais. Brasília: MS; 2004.
17. Luiz RR, Magnanini MF. A lógica da determinação do tamanho da amostra em investigações epidemiológicas. Cad Saude Colet 2000; 8(2):9-28.

18. Fleiss JL. Statistical methods for rates and proportions. New York: John Wiley Sons; 1981.

19. Cicchetti DV, Volkmar F, Sparrow SS, Cohen D, Fermanian J, Rourke BP. Assessing the reliability of clinical scales when the data have both nominal and ordinal features: proposed guidelines for europsychological assessments. J Clin Exp Neuropsychol 1992; 14(5):673-686.

20. World Health Organization (WHO). Oral health surveys: basic methods. $4^{\text {th }}$ ed. Geneva: ORH/EPID; 1997.

21. Oliveira BH, Nadanovsky P. Psychometric properties of the Brazilian version of the oral Health Impact Profile-Short form. Community Dent Oral Epidemiol 2005; 33(4):307-314.

22. Camelier AA. Avaliação da Qualidade de Vida Relacionada à Saúde em Pacientes com DPOC: estudo de base populacional com o SF-12 na cidade de São Paulo-SP [tese]. São Paulo: Universidade Federal de São Paulo; 2004.

23. Andersen RM, Davidson PL. Ethnicity, aging, and oral health outcomes: a conceptual framework. Adv Dent Res 1997; 11(2):203-209.

24. Locker D, Quinõnez C. To what extent do oral disorders compromise the quality of life? Community Dent Oral Epidemiol 2011; 39(1):3-11.

25. Martins AMEBL, Barreto SM, Pordeus IA. Características associadas ao uso de serviços odontológicos entre idosos dentados e edentados no Sudeste do Brasil: Projeto SBBrasil. Cad Saude Publica 2008; 24(1):81-92.

26. Mallmann FH, Toassi RFC, Abegg C. Perfil epidemiológico do uso e necessidade de prótese dentária em indivíduos de 50-74 anos de idade, residentes em três Distritos Sanitários de Porto Alegre, Estado do Rio Grande do Sul, Brasil, em 2008. Epidemiol Serv Saúde 2012; 21(1):79-88.

27. Silva DD, Sousa MLR, Wada RS. Saúde bucal em adultos e idosos na cidade de Rio Claro, São Paulo, Brasil. Cad Saude Publica 2004; 20(2):626-631.

28. Martins AMEBL, Haikal DS, Pereira SM, Barreto SM. Uso de serviços odontológicos por rotina entre idosos brasileiros: Projeto SB Brasil. Cad Saude Publica 2008; 24(7):1651-1666.

29. Rihs LB, Silva DD, Sousa MLR. Dental caries in an elderly population in Brasil. J Appl Oral Sci 2009; 17(1):8-12.

30. Roncalli AG, Côrtes MIS, Peres KG. Perfis epidemiológicos de saúde bucal no Brasil e os modelos de vigilância. Cad Saude Publica 2012; 28(Supl.):58-58.

31. Brasil. Ministério da Saúde (MS). Diretrizes da política nacional de saúde bucal. Brasília: MS; 2004.

32. Silva MÊS, Magalhães CS, Ferreira EF. Perda dentária e expectativa da reposição protética: estudo qualitativo. Cien Saude Colet 2010; 15(3):813-820.

33. Haikal DS, de-Paula AMB, Martins AMEBL, Moreira, AN, Ferreira EF. Autopercepção da saúde bucal e impacto na qualidade de vida do idoso: uma abordagem quanti-qualitativa. Cien Saude Colet 2011; 16(7):3317-3329. 
34. Goiato MC, Castelleoni L, Santos DM, Filho HG, Assunção WG. Lesões Orais Provocadas Pelo Uso de Próteses Removíveis. Pesq Bras Odontoped Clin Integr 2005; 5(1):85-90.

35. Vargas AMD, Paixão HH. Perda dentária e seu significado na qualidade de vida de adultos usuários de serviço público de saúde bucal do Centro de Saúde Boa Vista, em Belo Horizonte. Cien Saude Colet 2005; 10(4):1015-1024.

36. Maciel SSV, Souza RSV, Donato LMA, Albuquerque IGM, Donato LFA. Prevalência das Lesões de Tecidos Moles Causadas por Próteses Removíveis nos Pacientes da Faculdade de Odontologia de Caruaru, PE, Brasil. Pesq Bras Odontoped Clin Integr 2008; 8(1):93-97.

37. Vacarezza GF, Antunes JLF, Michaluart-Júnior PM. Recurrent sores by ill-fitting dentures and intra-oral squamous cell carcinoma in smokers. J Public Health Dent 2010; 70(1):52-57.

38. Ekbäck G, Astrøm AN, Klock K, Ordell S, Unell L. Variation in subjective oral health indicators of 65 -year-olds in Norway and Sweden. Acta Odontol Scand 2009; 67(4):222-232.

39. Petersen PE. The World Oral Health Report 2003: Continuous Improvement of Oral Health in the 21st Century-The Approach of the WHO Global Oral Health Programme. Geneva: World Health Organization; 2003.
40. Martins AMEBL, Jones KM, Souza JGS, Pordeus IA. Associação entre impactos funcionais e psicossociais das desordens bucais e qualidade de vida entre idosos. Cien Saude Colet 2014; 19(8):3461-3478.

41. Martins AMEBL, Barreto SM, Silveira MF, Santa-Rosa TTA, Pereira RD. Autopercepção da saúde bucal entre idosos brasileiros. Rev Saude Publica 2010; 44(5):912922.

42. Gerritsen AE, Finbarr Allen P, Witter DJ, Bronkhorst EM, Creugers NHJ. Tooth loss and oral health-related quality of life: a systematic review and meta-analysis. Health Qual Life Outcomes 2010; 8:126.

43. Özhayat EB. Influence of negative affectivity and self-esteem on the oral health related quality of life in patients receiving oral rehabilitation. Health Qual Life Outcomes 2013; 11:178.

Artigo apresentado em 20/02/2017

Aprovado em 29/01/2018

Versão final apresentada em 31/01/2018 